\title{
Les précipitations en 6 minutes. Premier examen de quelques mois d'enregistrement sur cassette magnétique
} Study of the initial 6 minutes of rainfall recordings

\author{
P. Guillot et D. Duband \\ Direction de la Production et du Transport \\ EDF - Grenoble
}

Nous avons commencé en 1980 à équiper tous nos pluviographes à auget basculeur, de l'enregistreur magnétique à cassette décrit dans la Houille Blanche (1975, $\mathrm{n}^{\circ} 4$, pp. 231-232). Nous utilisons ce matériel depuis plusieurs années pour enregistrer la température des cours d'eau dans une cinquantaine d'emplacements. (Houille Blanche, 1976, n 5, pp. 375-377).

Fin 1980, 30 pluviographes à auget étaient équipés, 80 le seront fin 1981,150 fin 1982; ainsi que 150 limnigraphes.

Les cassettes sont relevées et lues une fois par mois; cette périodicité pourra éventuellement être portée dans l'avenir à 2 ou 3 mois, avec la surveillance quotidienne $\mathrm{du}$ fonctionnement par liaison téléphonique, qui permettra de collecter directement les données horaires. Pour le moment, l'enregistrement graphique à relevé hebdomadaire est conserve" (du moins dans les emplacements où un observateur peut encore s'en charger) pour le contrôle visuel du bon fonctionnement $\mathrm{du}$ capteur.

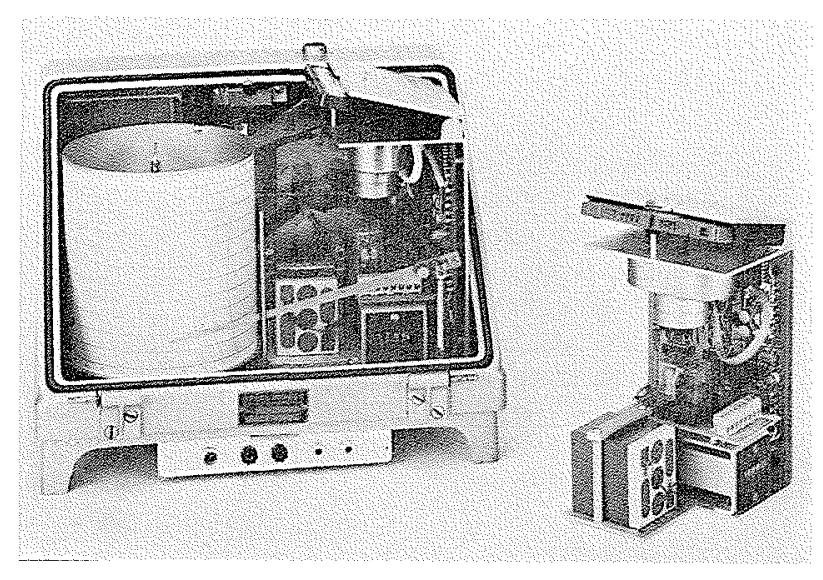

Figure 1 - Pluviographe équipé d'un enregistreur à cassette.
Nous rappelons que ce matériel (Fig. 1)

- résout économiquement le problème de l'enregistrement de longue durée sans intervention, dans les emplacements isolés et non (ou mal) desservis en énergie électrique ;

- permet l'automatisation de l'archivage informatique des précipitations à fine échelle de temps : 6 minutes, ou même aussi facilement 1 minute, et ceci avec une chronologie rigoureuse (les bandes graphiques hebdomadaires donnent laborieusement des données horaires, avec une incertitude d'une demi-heure ...).

Mais, diront certains, à quoi peut servir la connaissance des précipitations en 6 minutes ? Le pas de temps d'une heure n'est-il pas suffisant pour l'hydrologie? Citons trois applications qui n'épuisent pas le sujet, loin de là :

\section{Pluies extrêmes}

Analyse de la structure chronologique fine des averses pour savoir quelle réalité élémentaire recouvre l'hypothèse schématique du Gradex saisonnier des pluies horaire et journalière. Et si 3 à 5 ans de bonnes données "6 minutes" dans une station suffisaient à y caractériser le régime saisonnier des relations intensitédurée-fréquence, aussi bien, sinon mieux, que des dizaines d'années de pluies joumalières et horaires de qualité médiocre ? La rentabilité de ces données irait bien au-delà du ruissellement urbain.

\section{Relation pluie-débit}

Quelle est l'influence sur le coefficient d'écoulement immédiat, de l'irrégularité chronologique, et de l'irrégularité spatiale dont elle est l'indice ? Il n'est évidemment pas question de tracer des isohyètes décihoraires, seulement d'avoir un indice de concentration dans le temps et dans l'espace, génératrice de saturation du sol, facteur décisif de l'écoulement. 

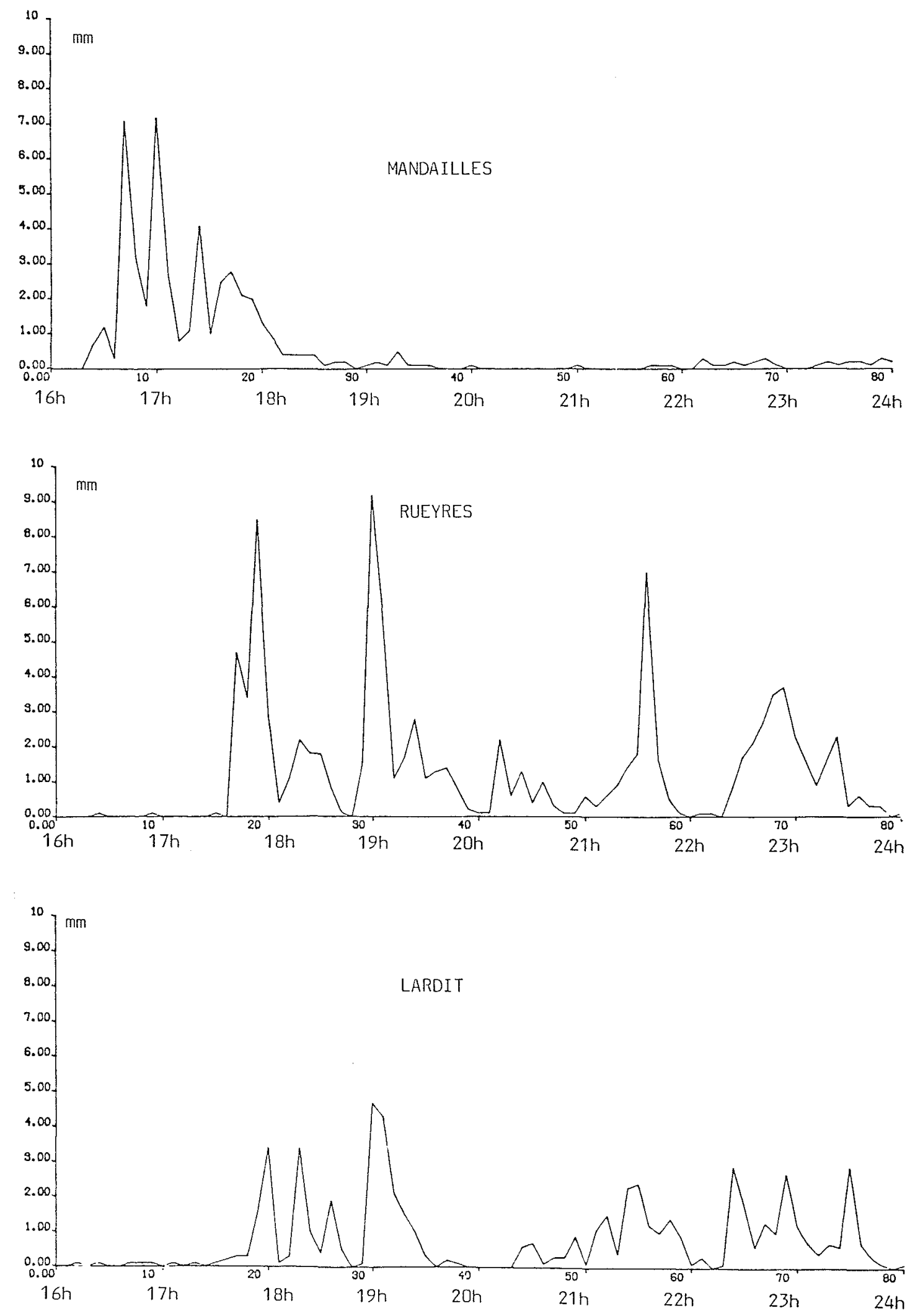

Figure 2 - Episode du 21 septembre 1980. 
Tableau I - Tableau de la pluie horaire en $1 / 10 \mathrm{~mm}$ calculée sur une journée $(8 \mathrm{~h}-8 \mathrm{~h}) 3$ épisodes pluvieux

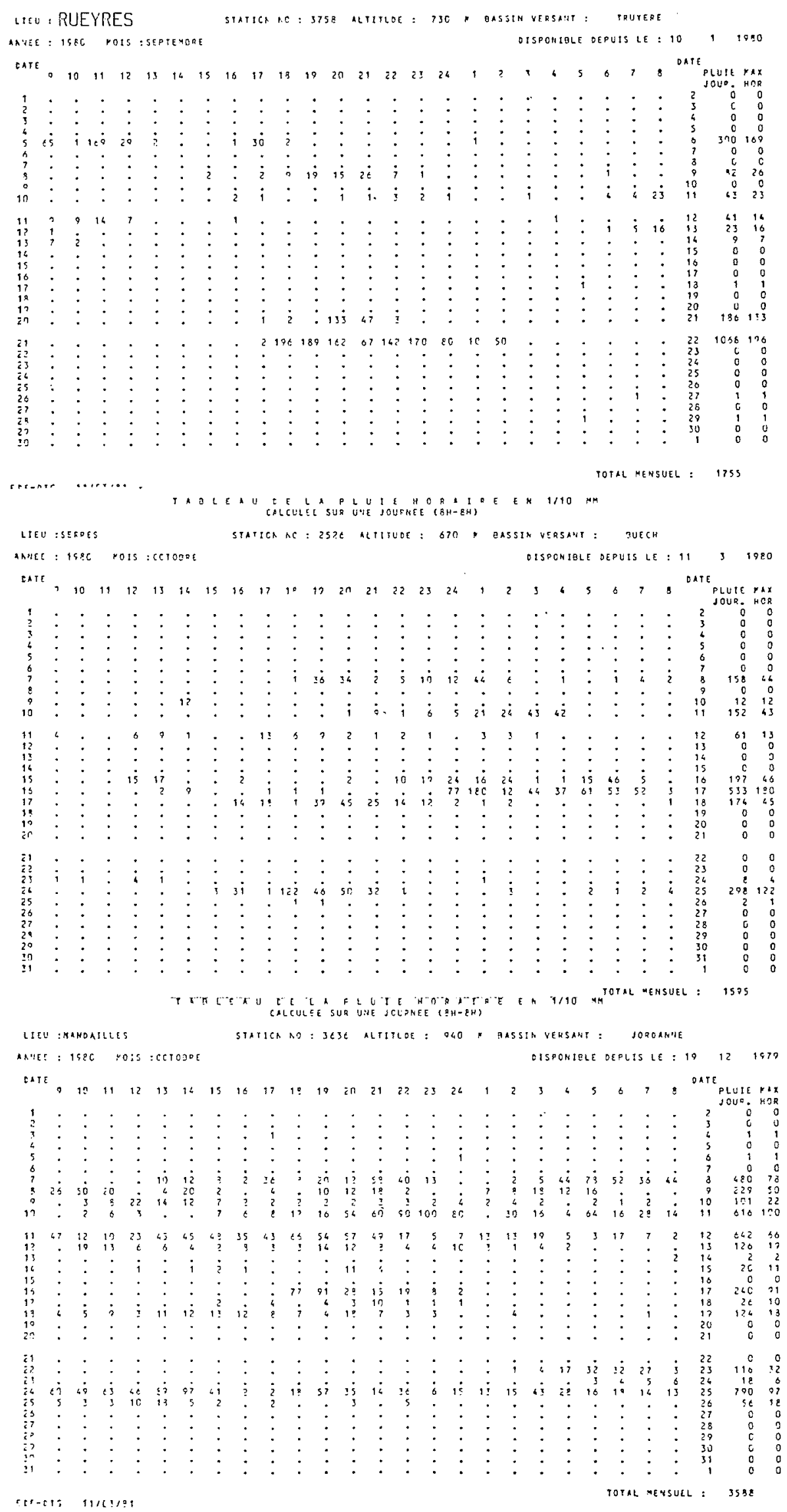




\section{Rectification des averses intenses}

Le basculement de l'auget présente un temps mort inévitable de 0,4 seconde, soit une perte de $7 \%$ quand l'intensité est d'un millimètre par minute, de $20 \%$ à $3 \mathrm{~mm} /$ minute. Malgré ce défaut, l'auget symétrique reste le principe de capteur de loin le plus avantageux.

Tous les dispositifs correcteurs (laminage du débit, clapet, électro-vanne, auget à poche d'air, palette cinétique,) sont insuffisants et infidèles. Le plus sage est de renoncer à ces perfectionnements, sources d'ennuis, et d'appliquer, au moment du traitement, une correction forfaitaire liée à l'intensité, qui sera d'autant mieux estimée que le temps d'intégration sera court.
Quelques résultats statistiques sur les mesures enregistrées du $1^{\text {er }}$ septembre au 31 décembre 1980

Nous avons représenté graphiquement (Fig. 2, 4,5) la chronologie temporelle, avec une image spatiale simplifiée, de trois épisodes pluvieux assez typiques (exemple des valeurs horaires, Tableau I) :

- l'averse du 21 septembre limitée à quelques heures et à faible extension spatiale en face sud du Cantal, mais très intense localement puisque des intensités en 6 minutes ont atteint $10 \mathrm{~mm}$ (stations de Mandailles, St-Etienne-Cantalès, Lardit, Rueyres, Aubrac, Grandval), et ont engendré une crue rapide sur la Bromme, avec dégats importants (Fig. 2 et 3 );

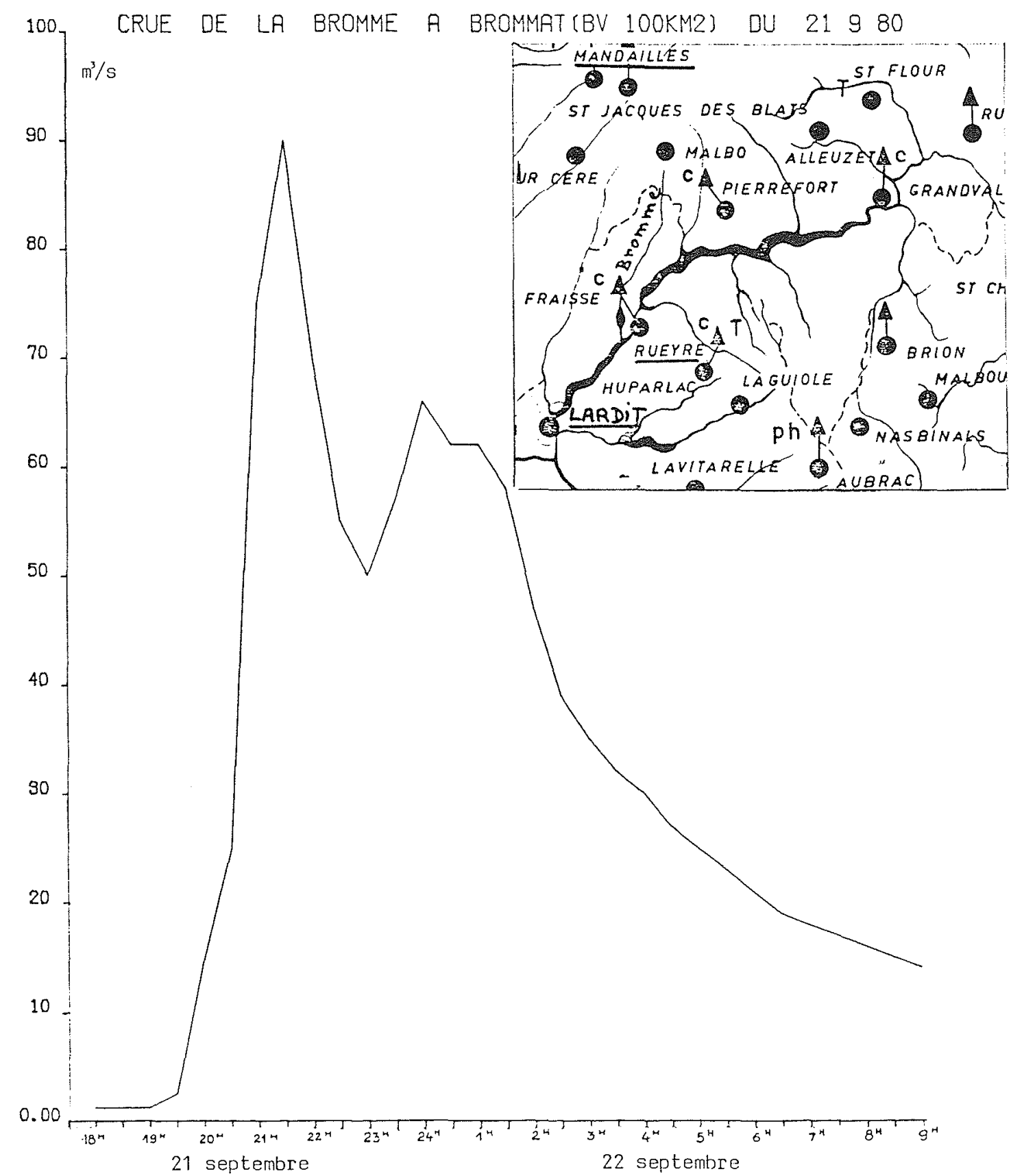

Figure 3 - Crue de la Bromme à Brommat (BV $100 \mathrm{~km}^{2}$ ) du 21 septembre 1980. 

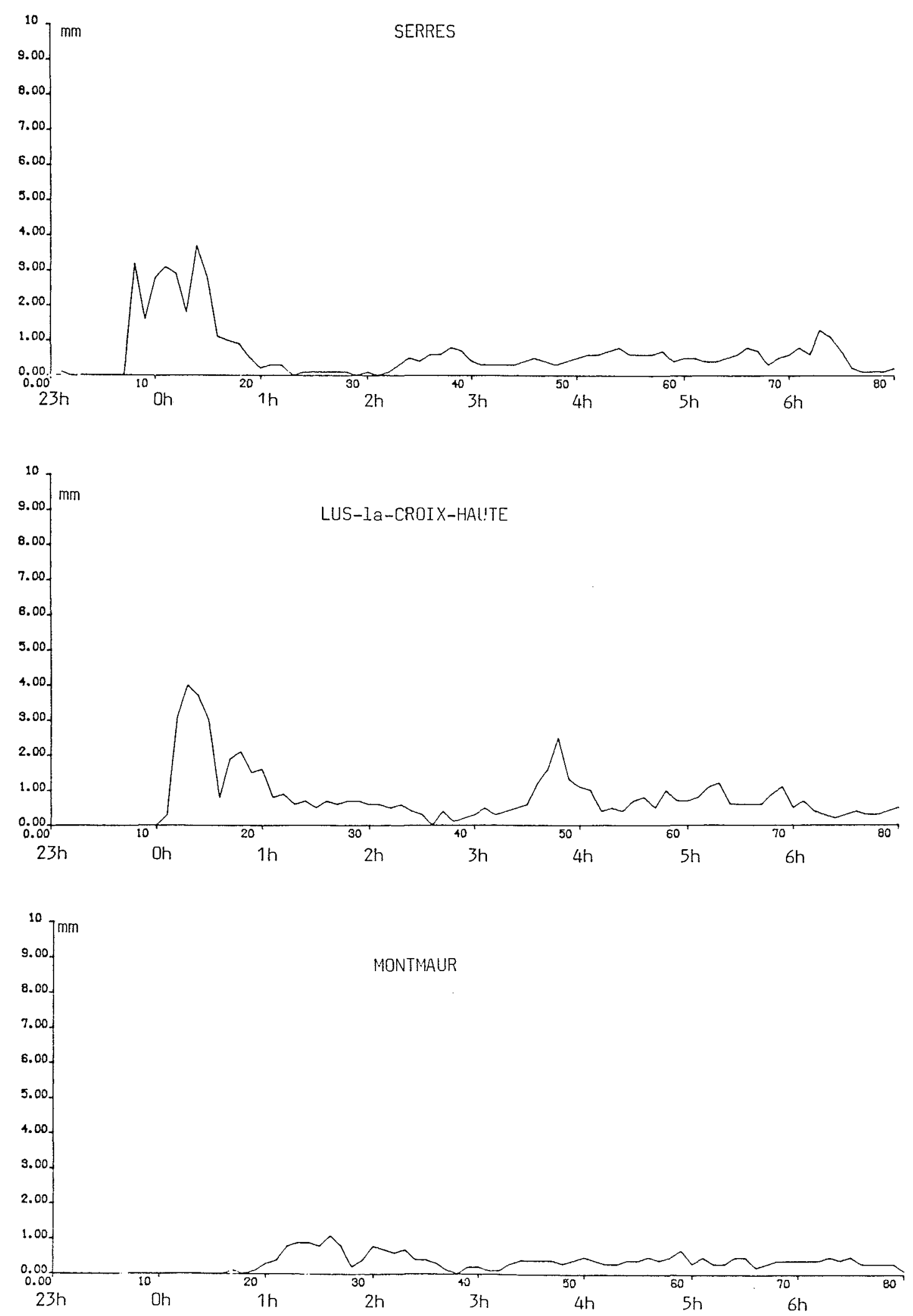

Figure 4 - Episode du 17 octobre 1980 (BV Buech). 

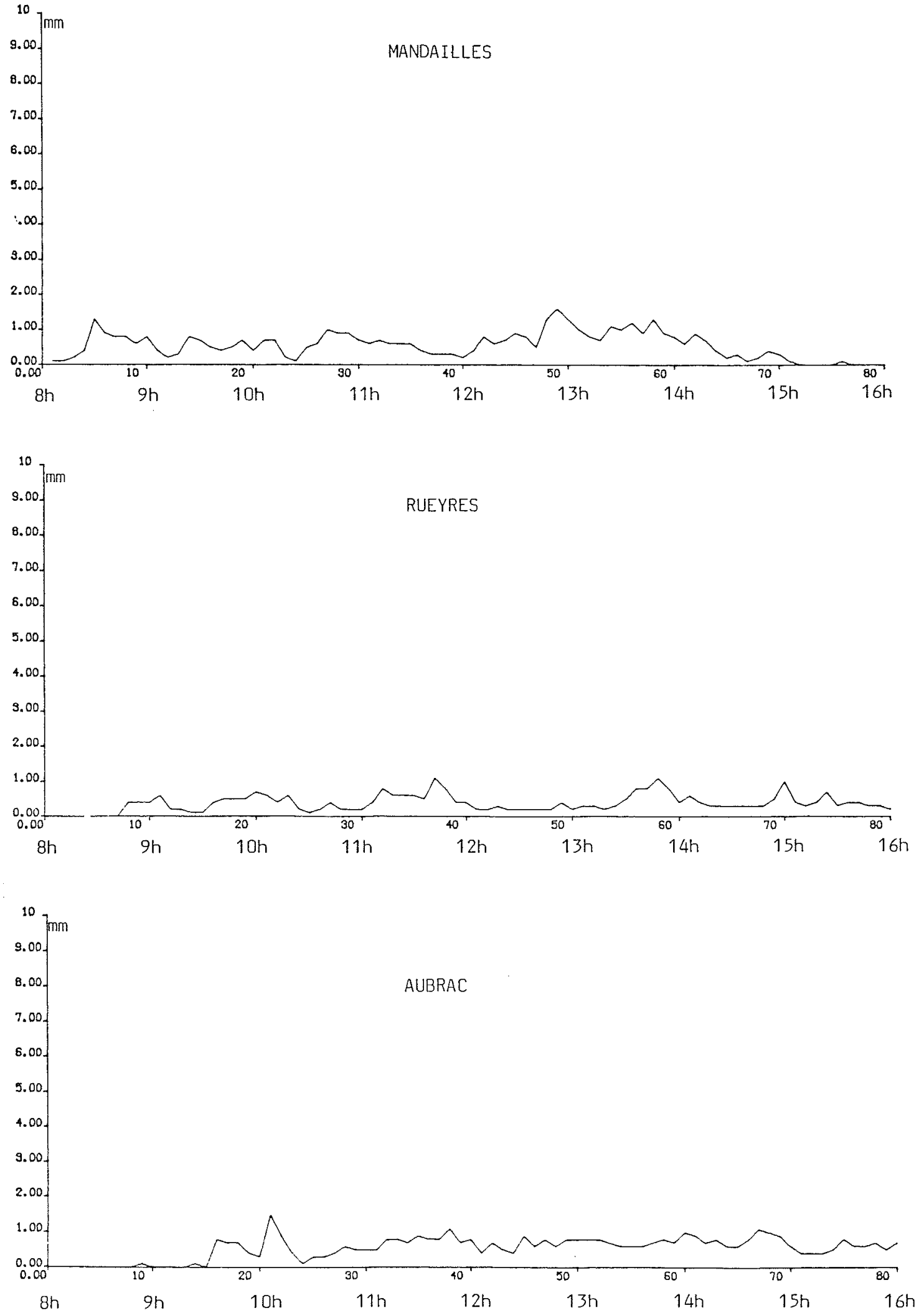

Figure 5 - Episode du 24 octobre 1980. 
- l'averse du 16 octobre, était relativement courte mais plus étendue dans les Alpes du Sud, avec des intensités locales moindres puisqu'elles n'ont pas dépassé $4 \mathrm{~mm}$ en 6 minutes (stations de Serres, Lus-la-CroixHaute, Montmaur, St-Etienne-en-Dévoluy) ; cet épisode, malgré son volume cumulé important, a été à l'origine d'une crue modeste sur le Buech, du fait de la faible humidification antérieure, donc peu de rendement du sol, et parce que l'averse s'est beaucoup atténuée en altitude dans le haut bassin (Fig. 4);

- une averse de longue durée le 24 octobre dans l'ouest du Massif-Central, mais dont les intensités sont modérées, les intensités en 6 minutes n'ont pas dépassé $1,5 \mathrm{~mm}$ (stations de Mandailles, St-Etienne-Cantalès, Rueyres, Lardit, Aubrac); cumulé sur trois jours, le volume de l'averse est assez important (Fig. 5).

\section{Analyse statistique effectuée sur 4 séries d'enregistements locaux}

- Fréquence des pluies au pas de temps de 6 minutes:

\begin{tabular}{|l|c|c|c|}
\hline & $\begin{array}{c}\text { Pluie } \\
\text { nulle }\end{array}$ & $\begin{array}{c}\text { Pluie } \\
<1 \mathrm{~mm}\end{array}$ & $\begin{array}{c}\text { Pluie } \\
>2 \mathrm{~mm}\end{array}$ \\
\hline Les Rousses (Jura) & $90.9 \%$ & $9 \%$ & $\cdot 1 \%$ \\
Serres (Alpes du Sud) & $95.8 \%$ & $4 \%$ & $\cdot 2 \%$ \\
Rueyres (Massif Central) & $93 \%$ & $6.7 \%$ & $\cdot 3 \%$ \\
Gedre (Pyrénées) & $86.3 \%$ & $13 \%$ & $\cdot 7 \%$ \\
\hline
\end{tabular}

Chiffres à comparer avec la fréquence des pluies nulles en 24 heures et en 1 heure pour la même période :
- Fréquence des pluies nulles en :

\begin{tabular}{|l|c|c|}
\hline & 1 heure & 24 heures \\
\hline Les Rousses & $76 \%$ & $38 \%$ \\
Serres & $90.9 \%$ & $70 \%$ \\
Rueyres & $86 \%$ & $59 \%$ \\
Gèdre & $76 \%$ & $45 \%$ \\
\hline
\end{tabular}

- Fréquence des séquences pluvieuses suivant leur longueur:

\begin{tabular}{|l|c|c|c|c|c|}
\hline & $\begin{array}{c}\text { Nombre } \\
\text { de } \\
\text { séquences }\end{array}$ & $\begin{array}{c}\text { Nombre } \\
\text { séquences } \\
\text { de 6 }\end{array}$ & $\begin{array}{c}\text { Longueur } \\
\text { moyenne }\end{array}$ & $\begin{array}{c}\text { Longueur } \\
\text { maximale } \\
\text { observée }\end{array}$ & $\begin{array}{c}\text { Ecart- } \\
\text { type }\end{array}$ \\
\hline Les Rousses & 765 & 471 & $21^{\prime}$ & $450^{\prime}$ & $43^{\prime}$ \\
Serres & 256 & 151 & $22^{\prime}$ & $294^{\prime}$ & $39^{\prime}$ \\
Ruevres & 407 & 239 & $23^{\prime}$ & $648^{\prime}$ & $59^{\prime}$ \\
Gèdre & 782 & 492 & $21^{\prime}$ & $906^{\prime}$ & $52^{\prime}$ \\
\hline
\end{tabular}

La longueur moyenne des séquences a peu varié au cours du second trimestre 1980.

\section{- Corrélogrammes calculés sur une demi-douzaine d'épisodes pluvieux continus, en précipitations horaires:}

Entre deux intervalles pluvieux de 6', l'autocorrélation se situe à environ . 8 ; entre deux pluies de $6^{\prime}$, séparées par 90 minutes, le coefficient d'autocorrélation se situe entre $\cdot 30$ et $\cdot 40$, et entre $\cdot 10$ et $\cdot 20$ pour deux pluies séparées de 180 minutes.

\section{Discussion}

\section{Le Président. - Je remercie M. GULLLOT.}

$\mathrm{Y}$ a-t-il des questions à poser ?

M. GHIO, - Quels sont les taux de pannes que vous observez sur les enregistrements?

M. GUILLOT. $-90 \%$ des cassettes mensuelles n'ont aucun défaut ; $10 \%$ ont quelques mesures invalidées par des parasites, mais généralement ces mesures peuvent être reconstituées, au total le taux de perte est très inférieur à $1 \%$.

Je suis confus de vous présenter ce matériel en service seulement en 1981, car il aurait du l'être depuis 8 ans; nous avons cru bien faire d'intéresser un grand constructeur, cela a abouti à un prix de commercialisation abusif, inacceptable, pour les applications hydrologiques. Pour nous, cela a donc été une perte de temps.

M. PERSOONS. - D'accord sur le principe suivant : enregistrement local le plus fin possible car ne coute pas cher et télémesure pour le temps réel et la télésurveillance du réseau.

Question : Avez-vous pu éliminer tous les parasites (orage et centrale électrique) tant sur les comptages que les bases de temps.

M. GUILLOT - En effet, il y a des parasites qui ont la propriété de remettre les mémoires à 0 , mais ce n'est pas très grave. Comme, de toute façon, notre système d'enregistrement est cumulatif, il y a un pas de temps qui est perdu, et comme le pas de temps est petit, le risque est limité.

Je rejoins tout à fait votre remarque sur la question de la surveillance. Actuellement, nous maintenons l'enregistrement graphique à côté des cassettes, parce qu'il permet de savoir d'un coup d'oil si le capteur a bien marché. C'est là l'étape actuelle. Dans l'avenir, la collecte par téléphone des valeurs horaires, mémorisées dans un répondeur placé à côté de chaque station, permettra la mise à jour quotidienne des données informatisées et leur visualisation sur écran-clavier. On pourra alors supprimer les enregistrements graphiques.

Le Président. - Il nous faut arrêter ici cette discussion parce que nous avons encore à entendre deux communications importantes. 


\section{Abstract}

\section{Study of the initiial 6 minutes of rainfall recordings}

The progressive installation of a cassette tape recorder in the EDF network of rainfall recorders (150 units of apparatus) :

- solves economically the problem of long recording (one to two months) in remote places, and

- allows for the automation of computerised files on rainfall in six minute time steps

The knowledge of rainfall using so small a time unit has a wide range of uses, including among other things, analysis of :
- the fine chronological structure of showers to examine more thoroughly the Gradex hypothesis adopted for extreme rainfall conditions, over a few hours or for a whole day; -.. the influence of the chronological irregularity of showers on the instantaneous flow coefficient in the rain/flow relation ;

- measurement quality improvement by allowing for an adjustment linked to intensity to offset the inherent loss of the recorder.

Examples are given of the processing of recent recordings. 